\title{
-NOTES-
}

\section{A NOTE ON THE SIMPLY-SUPPORTED PLATE*}

\section{BY M. STIPPES (Virginia Polytechnic Institute)}

The deflection of a simply-supported plate subjected to a concentrated load at some interior point is well-known in the form of a bilinear series [1] ${ }^{* *}$. In a recent paper, B. D. Aggarwala [2] has given closed expressions for the vertical shears $Q_{x}$ and $Q_{y}$ in terms of Weierstrassian elliptic functions $\wp(z)$ and their derivatives. The purpose of this note is to show how his results can be extended directly to give closed expressions for the bending moments $M_{x}, M_{\nu}$ and for the resultant vertical shears $V_{x}, V_{\nu}$ at the boundary of the plate.

We begin by considering a rectangular plate the mid surface of which is oriented as shown in the Argand diagram of Fig. 1. If the plate is subjected to a concentrated

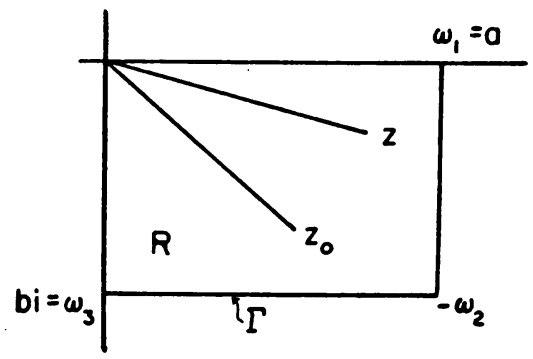

Fia. 1.

load $P$ at the point $z_{0}$ and is simply-supported on the boundary $\Gamma$, then the transverse deflection, $w(x, y)$, is determined by the following conditions.

$$
\nabla^{4} w=0 \text { in } R ; \quad w=0, \quad \nabla^{2} w=0 \text { on } \Gamma,
$$

and, additionally,

$$
w-\frac{P}{16 \pi D}\left(z-z_{0}\right)\left(z^{*}-z_{0}^{*}\right) \log \left(z-z_{0}\right)\left(z^{*}-z_{0}^{*}\right)=B_{1}\left(z, z_{0}, z^{*}, z_{0}^{*}\right),
$$

where $B_{1}$ is analytic in $R, z^{*}$ denoting the conjugate of $z$.

The physical interpretation of the condition expressed by Eq. (2) is nothing more than the fact that the singular part of $w$ in $R$ is such that the vertical shear caused by this deflection, when summed over the lateral surface of a cylinder of radius $\epsilon$ drawn about $z_{0}$ is equivalent to a load $P$. With $w$ so determined, the bending moments, vertical shears, and resultant vertical shears are computed by the usual expressions

*Received December 14, 1954; Revised manuscript received April 15, 1955.

**Numbers in square brackets refer to the bibliography at the end of this paper. 


$$
\begin{array}{ll}
M_{x}=-D\left(\frac{\partial^{2} w}{\partial x^{2}}+\sigma \frac{\partial^{2} w}{\partial y^{2}}\right) & M_{y}=-D\left(\frac{\partial^{2} w}{\partial y^{2}}+\sigma \frac{\partial^{2} w}{\partial x^{2}}\right) \\
Q_{x}=-D \frac{\partial}{\partial x} \nabla^{2} w & Q_{y}=-D \frac{\partial}{\partial y} \nabla^{2} w \\
V_{x}=-D \frac{\partial}{\partial x}\left[\frac{\partial^{2} w}{\partial x^{2}}+(2-\sigma) \frac{\partial^{2} w}{\partial y^{2}}\right] & \\
V_{y}=-D \frac{\partial}{\partial y}\left[\frac{\partial^{2} w}{\partial y^{2}}+(2-\sigma) \frac{\partial^{2} w}{\partial y^{2}}\right] . &
\end{array}
$$

Now in Eq. (1), we let $\nabla^{2} w=M$ and find that

$$
\nabla^{2} M=0 \text { in } R ; \quad M=0 \text { on } \Gamma
$$

where $M$ is subject to the condition that

$$
M-\frac{P}{4 \pi D} \log \left(z-z_{0}\right)\left(z^{*}-z_{0}^{*}\right)
$$

is analytic in $R$. Now an elementary application of the Shwartz-Christoffel mapping theorem along with the method of images yields

$$
\nabla^{2} w=\frac{P}{4 \pi D} \log \left\{\frac{\left[\wp(z)-\wp\left(z_{0}\right)\right]\left[\wp\left(z^{*}\right)-\wp\left(z_{0}^{*}\right)\right]}{\left[\wp\left(z^{*}\right)-\wp\left(z_{0}\right)\right]\left[\wp(z)-\wp\left(z_{0}^{*}\right)\right]}\right\} .
$$

The function $\wp(u)$ is the Weierstrassian elliptic function with periods $2 \omega_{1}, 2 \omega_{3}$. (This result, of course, is well-known.)

From Eq. (4), it follows that $w$ can be written in the form

$$
\begin{aligned}
w(x, y)=\frac{P}{16 \pi D}\left(z-z_{0}\right)\left(z^{*}-z_{0}^{*}\right) \log \left\{\frac{\left[\wp(z)-\wp\left(z_{0}\right)\right]\left[\wp\left(z^{*}\right)-\wp\left(z_{0}^{*}\right)\right]}{\left[\wp\left(z^{*}\right)-\wp\left(z_{0}\right)\right]\left[\wp(z)-\wp\left(z_{0}^{*}\right)\right]}\right\} \\
+\frac{P}{16 \pi D} B\left(z, z_{0}, z^{*}, z_{0}^{*}\right),
\end{aligned}
$$

where the function $B$ is analytic inside the rectangle, $R$, vanishes on the boundary $\Gamma$, and satisfies the following Poisson equation in $R$

$$
\frac{\partial^{2} B}{\partial z \partial z^{*}}=-\left(z^{*}-z_{0}^{*}\right)\left[\frac{\wp^{\prime}\left(z^{*}\right)}{\wp\left(z^{*}\right)-\wp\left(z_{0}^{*}\right)}-\frac{\wp^{\prime}\left(z^{*}\right)}{\wp\left(z^{*}\right)-\wp\left(z_{0}\right)}\right]+\text { complex conjugate. }
$$

Now Eq. (4) is one linear relation between the derivatives $w_{x x}$ and $w_{y y}$. One more relation between these two quantities, independent of Eq. (4), will permit their explicit presentation. To this end, we note that in view of the definitions of $Q_{x}$ and $Q_{y}$ along with Eq. (4) both $Q_{x}$ and $Q_{y}$ are harmonic functions. Consequently, there results from Eq. (3b) the following relation

$$
\nabla^{2}\left(\square^{2} w\right)=-\frac{1}{D}\left(\frac{\partial Q_{x}}{\partial x}-\frac{\partial Q_{\nu}}{\partial y}\right), \quad \square^{2} \equiv \frac{\partial^{2}}{\partial x^{2}}-\frac{\partial^{2}}{\partial y^{2}} .
$$

Furthermore, since

$$
\nabla^{2}\left(\frac{x}{2} Q_{x}\right)=\frac{\partial Q_{x}}{\partial x}, \quad \nabla^{2}\left(\frac{y}{2} Q_{\nu}\right)=\frac{\partial Q_{y}}{\partial y}
$$


Eq. (7) may be rewritten as

$$
\nabla^{2}\left\{\square^{2} w+\frac{1}{2 D}\left(x Q_{x}-y Q_{v}\right)\right\}=0 .
$$

It should be remarked that this is the key relationship for the determination of the desired quantities.

From Eq. (9), we have

$$
\square^{2} w+\frac{1}{2 D}\left(x Q_{x}-y Q_{y}\right)=H_{1}(x, y),
$$

where $H_{1}$ is a harmonic function. To determine $H_{1}$, uniquely, it is necessary to know its boundary values, and, additionally, the nature of its singularity in $R$. For a convenience in a future integration, we shall determine, instead of $H_{1}$, the function

$$
I_{1}(x, y)=H_{1}(x, y)+\frac{\omega_{3}}{2 D i} Q_{y}-\frac{\omega_{1}}{2 D} Q_{x} .
$$

Since the plate is simply-supported at the boundary, we have

$$
w=0, \quad \nabla^{2} w=0 \text { on } \Gamma \quad \text { implying } \quad \square^{2} w=0 \text { on } \Gamma .
$$

The boundary values of $I_{1}$ are then determined from Eq. (10) and Eq. (11). An investigation of Eq. (5) reveals the nature of the singular part of $I_{1}$.

From the addition theorem for the $\wp$ functions, it follows that $\wp(z)=\varnothing\left(z^{*}\right)$ anywhere on $\Gamma$. With this observation, the following three functions

$$
\begin{aligned}
& R_{1}=\frac{P}{8 \pi D}\left[\frac{z_{0}^{*} \wp^{\prime}\left(z_{0}\right)}{\wp(z)-\wp\left(z_{0}\right)}-\frac{z_{0}^{*} \wp^{\prime}\left(z_{0}\right)}{\wp\left(z^{*}\right)-\wp\left(z_{0}\right)}\right]+\text { complex conjugate } \\
& R_{2}=\frac{P}{4 \pi D}\left[\frac{\wp^{\prime}\left(z_{0}\right)}{\wp(z)-\wp\left(z_{0}\right)}-\frac{\wp^{\prime}\left(z_{0}\right)}{\wp\left(z^{*}\right)-\wp\left(z_{0}\right)}\right]+\text { complex conjugate } \\
& R_{3}=R_{2} i
\end{aligned}
$$

(considered as functions of $z$ with $z_{0}$ as a parameter) vanish on $\Gamma$. Additionally, each of these functions is a harmonic function with such type singularity in $R$ that

$$
J_{1}(x, y)=H_{1}(x, y)+R_{1}+\frac{\omega_{3}}{2 D i}\left(Q_{y}+R_{3}\right)-\frac{\omega_{1}}{2 D}\left(Q_{x}+R_{2}\right)
$$

is analytic in $R$. Evidently $J_{1}$ assumes the same boundary values on $\Gamma$ as does $I_{1}$.

Since Green's function for LaPlace's equation for a rectangular region is known, we have

$$
J_{1}=\int_{\Gamma} I_{1} \frac{\partial G}{\partial n} d s
$$

If now we perform the indicated integration in Eq. (15), then after some laborious computations there results

$$
\begin{aligned}
F \equiv \frac{1}{2} H_{1}(x, y)-\frac{1}{4 D}\left(x Q_{x}-y Q_{y}\right) \\
=\frac{-i}{8 \pi D}\left[4 \omega_{1} \omega_{3} \zeta(z)-2\left(\eta_{1} \omega_{3}+\omega_{1} \eta_{3}\right) z-\pi i z^{*}\right]\left[Q_{x}\left(z, z_{0}\right)-i Q_{\nu}\left(z, z_{0}\right)\right] \\
\quad-\frac{i}{8 \pi D}\left[4 \omega_{1} \omega_{3} \zeta\left(z_{0}\right)-2\left(\eta_{1} \omega_{3}+\omega_{1} \eta_{3}\right) z_{0}-\pi i z_{0}^{*}\right]\left[Q_{x}\left(z_{0}, \mathrm{~s}\right)-i Q_{v}\left(z_{0}, z\right)\right] \\
\quad+\text { complex conjugate, }
\end{aligned}
$$


where by definition

$$
\zeta\left(\omega_{1}\right)=\eta_{1}, \quad \zeta\left(\omega_{3}\right)=\eta_{3} .
$$

(It is to be noted that $\zeta(z)$ is the Weierstrassian function associated with $\wp(z)$.)

This completes the analysis, for from Eqs. (4) and (10) it follows that

$$
\begin{aligned}
& w_{x x}=\frac{1}{2} \nabla^{2} w+F, \\
& w_{\nu y}=\frac{1}{2} \nabla^{2} w-F,
\end{aligned}
$$

and, consequently, all of the expressions in Eqs. (3) can now be presented in closed form.

If we introduce Jacobi's theta functions, we can write

$$
\begin{aligned}
F=\frac{P i}{8 \pi^{2} D}\left[\frac{\omega_{3}}{\omega_{1}} \frac{\theta_{1}^{\prime}(\nu)}{\theta_{1}(\nu)}+\pi i\left(\nu-\nu^{*}\right)\right] \frac{\theta_{1}(\nu) \theta_{2}(\nu) \theta_{3}(\nu) \theta_{4}(\nu) \theta_{1}\left(\nu_{0}+\nu_{0}^{*}\right) \theta_{1}\left(\nu_{0}-\nu_{0}^{*}\right)}{\theta_{1}\left(\nu+\nu_{0}\right) \theta_{1}\left(\nu-\nu_{0}\right) \theta_{1}\left(\nu+\nu_{0}^{*}\right) \theta_{1}\left(\nu-\nu_{0}^{*}\right)} \\
+\frac{P i}{8 \pi^{2} D}\left[\frac{\omega_{3}}{\omega_{1}} \frac{\theta_{1}^{\prime}\left(\nu_{0}\right)}{\theta_{1}\left(\nu_{0}\right)}+\pi i\left(\nu_{0}-\nu_{0}^{*}\right)\right] \frac{\theta_{1}\left(\nu_{0}\right) \theta_{2}\left(\nu_{0}\right) \theta_{3}\left(\nu_{0}\right) \theta_{4}\left(\nu_{0}\right) \theta_{1}\left(\nu+\nu^{*}\right) \theta_{1}\left(\nu-\nu^{*}\right)}{\theta_{1}\left(\nu_{0}+\nu\right) \theta_{1}\left(\nu_{0}-\nu\right) \theta_{1}\left(\nu_{0}+\nu^{*}\right) \theta_{1}\left(\nu_{0}-\nu^{*}\right)} \\
\quad+\text { complex conjugate, }
\end{aligned}
$$

where

$$
\nu=\frac{z}{2 \omega_{1}}, \quad \nu_{0}=\frac{z_{0}}{2 \omega_{1}} .
$$

By some arguments involving the properties of the real and complex parts of analytic functions $z$ of a complex variable $z$, it is possible to show that

$$
w_{x y}+\frac{1}{4 D}\left(y Q_{x}+x Q_{y}\right)=-\frac{1}{2} K_{1}\left(z, z_{0}, z^{*}, z_{0}^{*}\right)+K\left(z_{0}, z_{0}^{*}\right),
$$

where $K_{1}$ is the harmonic conjugate of $H_{1}$. However, there seems to be no immediate way of evaluating the function $K$.

\section{REFERENCES}

1. S. Timoshenko, Theory of plates and shells, McGraw-Hill Book Co., New York and London, 1940, p. 122

2. B. D. Aggarwala, Singularly loaded rectangular plates, Z. angew. Math. Mech. 34, No. 6 (1954)

3. E. T. Whittaker and G. N. Watson, Modern analysis, Fourth Ed., Cambridge, 1927

\section{GRAPHICAL DETERMINATION OF A DISCONTINUITY SURFACE BY WAVE REFLECTION*}

\section{BY HEINZ G. HELFENSTEIN (University of Alberta)}

1. Introduction. The reflection of waves, such as seismic reflection for prospecting has been discussed in many papers and books. The methods used in practice all share the simplification that the discontinuity surface is replaced in the neighborhood of the shot point by its tangent plane, and it is only this tangent plane which is determined. We shall discuss here a mathematically exact and fairly simple graphical method of

-Received Jan. 26, 1955; revised manuscript received April 18, 1955. 